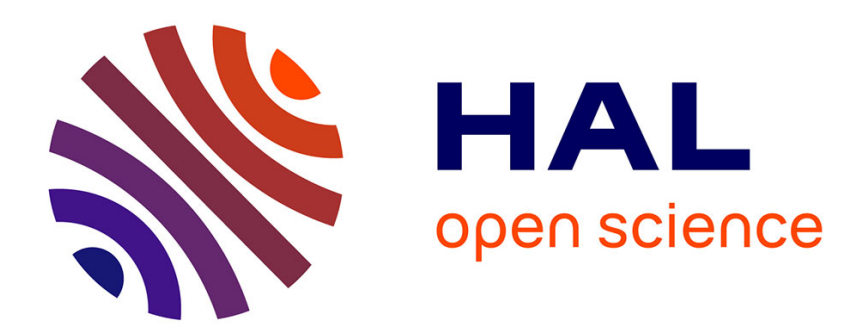

\title{
A model for the evolution of crèching behaviour in gulls
} Aurelien Besnard, Olivier Gimenez, Jean-Dominique Lebreton

\section{To cite this version:}

Aurelien Besnard, Olivier Gimenez, Jean-Dominique Lebreton. A model for the evolution of crèching behaviour in gulls. Evolutionary Ecology, 2002, 16 (5), pp.489-503. 10.1023/A:1020809528816 . hal03515254

\section{HAL Id: hal-03515254 \\ https://hal.science/hal-03515254}

Submitted on 6 Jan 2022

HAL is a multi-disciplinary open access archive for the deposit and dissemination of scientific research documents, whether they are published or not. The documents may come from teaching and research institutions in France or abroad, or from public or private research centers.
L'archive ouverte pluridisciplinaire HAL, est destinée au dépôt et à la diffusion de documents scientifiques de niveau recherche, publiés ou non, émanant des établissements d'enseignement et de recherche français ou étrangers, des laboratoires publics ou privés. 


\title{
A model for the evolution of crèching behaviour in gulls.
}

\author{
AURELIEN BESNARD ${ }^{1}$, OLIVIER GIMENEZ ${ }^{1}$ AND \\ JEAN-DOMINIQUE LEBRETON $^{1}$
}

1 - CEFE-CNRS, 1919 Route de Mende, 34293 Montpellier cedex 5

\begin{abstract}
Corresponding author : Aurélien Besnard
CEFE-CNRS

1919, Route de Mende

34293 Montpellier cedex 5

France

Tel : +33(0) 467613298

Fax : +33(0) 467412138

besnard@cefe.cnrs-mop.fr
\end{abstract}

Keywords : Crèching behaviour, ESS, Gulls, predation, habitat instability

Running headline : Crèching behaviour in Gulls 
3 Crèche is the aggregation of chicks outside the nesting territories in which chicks

4 continue to be fed only by their own parents. Several adaptive functions of crèching

5 have been proposed, the most frequent one being a reduction in predator pressure. Using

6 an evolutionary stable strategy approach based on the computation of individuals'

7 fecundity, we examined which regime of aerial and terrestrial predation is likely to

8 favour the evolution and stability of the crèching strategy in gulls. Our results confirm

9 the hypothesis that habitat instability associated with high levels of terrestrial predation

10 favours the evolution and maintenance of the crèching behaviour. Moreover, our results

11 suggest that a low aggressiveness against predators by offspring may be a pre-

12 adaptation to the crèching strategy. In contrast, the high synchronisation often observed

13 in crèching species does not favour the evolution of a crèching behaviour and is thus

14 probably under selection pressures different from those modelled here.

15

16

17

18 
3 Coloniality, defined as aggregation on a breeding site that is dissociated from foraging 4 sites (Wittenberger and Hunt, 1985; Siegel-Causey and Kharitonov, 1991) is a complex 5 reproductive strategy that brings many individuals simultaneously to a relatively small 6 breeding site. Although the evolution of coloniality has been intensively studied, the 7 factors responsible for its evolution and maintenance are still subject of much 8 discussion (see Danchin and Wagner, 1997 for a review). Coloniality is usually 9 associated with several life history traits such as collective anti-predator behaviour 10 (Wittenberger and Hunt, 1985; Siegel-Causey and Kharitonov, 1991), synchronisation 11 of the breeding activities (Gochfeld 1980) and a high frequency of aggressive interactions (Tinbergen 1956, 1959).

The formation of large groups of chicks in or near the colony site is one of the

14 most intriguing traits associated with coloniality. These groups are referred to as a 15 crèche, the crèching strategy being defined as an aggregation of chicks outside the 16 nesting territories in which the chicks continue to be fed only by their own parents. 17 Crèching has been reported in seven of the 14 colonial waterbirds families (A. Besnard and C. Tourenq, unpubl. results). However, the proportion of crèching species within these families varies greatly, ranging from a single crèching species (e.g., the banded 20 stilt, Cladorhynchus leucocephalus) in the Recurvirostridae (del Hoyo et al., 1996) to all 21 members of the family (e.g. flamingos) in the Phoenicopteridae (del Hoyo et al., 1996). Several functions of the crèching behaviour have been proposed, the most 23 frequently mentioned being the reduction of predation (Pettingill, 1960; Schaller, 1964; 24 Beer, 1966; Buckley and Buckley, 1972; Spurr, 1975; Evans, 1984; Carter and Hobson, 
1 1988). In a previous study of the crèching behaviour in gulls (Laridae, Larini following

2 Sibley and Ahlquist 1990), we demonstrated that crèching species all occupy unstable

3 habitats such as lagoon or river islets (Besnard et al. submitted). Such unstable habitats

4 are characterised by a high probability that colony sites become unsuitable following a

5 drying of the lagoon or a flooding of the breeding site. This instability of the habitat

6 could exert an important selective pressure on crèching behaviour and thus its evolution.

7 In non-crèching larids, the chicks usually remain on the nesting territories where

8 they are fed and protected against conspecifics by their parents until they fledge (del

9 Hoyo et al. 1996). In crèching species, the chicks leave the nesting territories only few

10 days after hatching and roam in the colony for a short period before they congregate in a

11 crèche at the periphery of the colony and possibly definitively leave the colony site

12 (Beer, 1966; Isenmann, 1976; del Hoyo et al., 1996). To explain this behaviour we

13 propose that crèching may allow for chicks to leave the colony site when it becomes

14 unsuitable for rearing chicks (flooded or accessible to terrestrial predators following the

15 drying of the water surrounding the colony) while maintaining the colony structure and

16 its ability to defend against predation (Besnard et al. submitted).

17 In order to test this hypothesis, in this paper we study the effect of a high

18 predation rate associated with a high probability for the colony site to become

19 accessible to terrestrial predation over the course of a single breeding season. An

20 Evolutionary Stable Strategy (ESS) approach is used. Individual fecundity was

21 computed using a reproductive output model based on cumulative predation risks over

22 time. We thus examined which characteristics of predation favour the evolution and the

23 maintenance of the crèching strategy. 
2 (Beer, 1966; Isenmann, 1976; Zubakin, 1985; Mierauska and Buzun, 1991), and are

3 described as being poorly aggressive against the predators of their chicks (Beer 1966,

4 Isenmann 1976, Veen 1977, Zubakin 1985), we focus on the effects of synchronisation

5 of the laying and anti-predator behaviour on the evolutionary stability of the crèching

6 strategy.

\section{THE MODELS}

9 All parameters used in the models are summarised in Table 1. We considered a colony 10 of approximately constant size (i.e. $N$ pairs) over the course of the breeding season.

11 We were only interested in the predators of chicks. We distinguished two different 12 phases of the breeding period, the incubation and the rearing period. Let $F(t)$ be the 13 cumulative distribution of laying dates, i.e. the fraction of the $N$ pairs which started to 14 breed before time $t$ and $m$ the individual reproductive output, assumed to be constant. 15 The number of eggs $\omega$ and chicks $\pi$ on the colony site at time $t$ are given by:

16 obtained as the sum of (1) and (2):

$$
\begin{aligned}
& \omega(t)=m N\left[F(t)-F\left(t-t_{i}\right)\right] \\
& \pi(t)=m N\left[F\left(t-t_{i}\right)-F\left(t-t_{b}\right)\right]
\end{aligned}
$$

where $t_{i}$ denote the duration of the incubation, $t_{r}$ is the rearing period and $t_{b}$ stands for the total time for which young were potential preys (from laying to fledging). The total number of items (eggs or chicks) in the colony at time $t$, denoted $n(t)$ is simply 


$$
n(t)=m N\left[F(t)-F\left(t-t_{b}\right)\right]
$$

3 Assuming that eggs and chicks have the same probability of being taken by a predator

4 and let $k$ be the instantaneous rate of predator attacks, the probability $p$ for an egg or a

5 chick to be preyed between time $t$ and $t+d t$ is given by:

6

$$
p=\frac{k}{n(t)} d t
$$

8 which reflects the dilution effect of the predation effort over $n(t)$ items present at time

$9 t$ (cf. selfish herd effect Hamilton, 1971).

10 Our model considers two kinds of predation: aerial and terrestrial. The aerial

11 predation rate, $k_{a}$, is assumed to be constant over the course of the breeding season.

12 Gulls, usually breeding on sites surrounded by water, are protected against terrestrial

13 predators. Terrestrial predation can only occur after the drying of colony periphery. The

14 occurrence of such a drying during the breeding season is more or less probable

15 according to the habitat characteristics occupied by a species (from highly probable in

16 temporary marsh or lagoon to highly improbable in permanent marsh or lake). Let $a$ be

17 the date at which the colony becomes accessible to terrestrial predation. The terrestrial

18 predation rate, denoted $k_{g}$, is null before $a$ and constant after $a$ until the end of the

19 breeding season (Model 1).

20 Gulls often actively defend the colony site against predators using "mobbing" 21 behaviour (Patterson, 1965; Kruuk, 1967; del Hoyo et al., 1996) which consists in 22 collective flights directed towards the predator. While mobbing has been demonstrated 23 to be an effective deterrent against aerial predators (reviewed in Wittenberger and Hunt, 
1 1985), its impact on terrestrial predator is often described as null (Veen 1977,

2 Wittenberger and Hunt 1985). In order to take the mobbing behaviour into account,

$3 \quad k_{a}$ is assumed to be a decreasing function of the number of breeding pairs still present at

4 time $t$ on the colony site (Model 2).

In order to retain the simplicity of the models, mortality is assumed not to affect

6 the number of eggs or chicks present on the breeding sites. We do not expected

7 qualitative results of the models to be changed by this assumption.

8

9 Crèching versus Non-crèching strategy

10 Chicks of non-crèching species stay on the colony site until they fledge

11 (Tinbergen, 1956; del Hoyo et al., 1996) and are preyed on by aerial and terrestrial

12 predators during the entire breeding season. In contrast, chicks of crèching species

13 usually leave the site when it becomes accessible to terrestrial predators and move to a

14 "safe" site (Beer, 1966; Isenmann, 1976; Zubakin and Flint, 1980; Besnard et al.,

15 submitted). We thus considered that the chicks of the crèching species remain at their

16 birth site as long as it remains protected against terrestrial predators. Contrary to the

17 non-crèching chicks, they move to a site protected against the terrestrial predators in the

18 immediate proximity of their birth site as soon as it becomes accessible. After having

19 left their birth site, chicks escape terrestrial predators but remain exposed to aerial

20 predators.

We examined the ecological conditions under which the Non-Crèching Strategy

22 (hereafter NCS) or the Crèching Strategy (CS) are ESS. If adult survival is equal for

23 two strategies A and B, strategy A is non-invasible by the strategy B when, in a

24 population fixed for $\mathrm{A}$, the fecundity of an $\mathrm{A}$ individual is greater than that of a B 
1 individual (Maynard Smith 1982). If only two strategies, A and B, are possible, the

2 study of invasibility of $\mathrm{A}$ is equivalent to the study of the evolutionary stability of A.

3 Strategy A is ESS if, in a population fixed for the strategy A, the ratio of the fecundity

4 of an individual exhibiting strategy A to that of an individual with strategy B is greater

5 than one.

6 The number of surviving offspring at fledging, later called fecundity, for a pair 7 laying at date $l$ according to the biological rules stated above was derived. The four 8 equations of fecundity were computed: (a) a NCS individual in a NCS population, (b) a

9 CS individual in a NCS population, (c) a NCS individual in a CS population and (d) a 10 CS individual in a CS population.

11 The fecundity of the four classes of individuals were derived for two cases: one 12 with $k_{a}$ constant (no active defence against predators: Model 1) and another one with $13 k_{a}$ a decreasing function of the breeding pairs on the colony site (active defence against 14 predators: Model 2). When there is no active defence against predators, the non15 crèching and crèching chicks suffer from the same aerial predation while only the noncrèching chicks suffer from terrestrial predation. Under these circumstances, CS always

17 outperforms NCS. The predictions from Model 1 are that crèching is an ESS as soon as 18 terrestrial predation is not null. However, we chose to present the computation of this 19 first model in order to progressively detail the different steps in our modelling approach.

20 When adults present an active defence against predators, the pay-off of each strategy is 21 affected by the number of chicks in the crèche or in the colony at all dates. Under these 22 circumstances, the modelling becomes crucial. Fecundity for both models are 23 respectively summarised in Tables 2 and 3 and more details are given in appendices A 24 and B. 
RESULTS

5

6

Model 1: without active defence

7

Table 2 approximately here

8

9 NCS is evolutionarily stable if the following inequality holds (Table 1 left part):

$$
\int_{a}^{l+t_{b}}-\frac{k_{g}}{n(t)} d t>0
$$

12 Inequality (5) has obviously no solution in our ecological situations because $k_{g}$ is

13 positive or zero and $n(t)$ is strictly positive whatever $t$. Hence, NCS is always invasible 14 by CS.

15

16 CS is evolutionarily stable if the following inequality holds (Table 2 right part):

$$
\int_{a}^{l+t_{b}} \frac{k_{g}}{\omega(t)} d t>0
$$

19 Inequality (6) is satisfied in several ecological situations. However CS is invasible by

20 NCS if the number of egg laid in the colony was infinite $(\omega(t)$ infinite) but this situation

21 is biologically uninteresting. CS is invasible by NCS if the rate of terrestrial attacks is 
1 zero $\left(k_{g}=0\right)$ or when the site becomes accessible to the terrestrial predators only when

2 or after the chick have fledged $\left(a \geq l+t_{b}\right)$.

3 To sum up, CS is evolutionarily stable if the actual terrestrial predation on the 4 chicks is not zero. On the contrary, NCS is always invasible by CS. As a consequence, 5 the CS is convergent as soon as the realised terrestrial predation on the chicks is not

6 zero. In contrast, if terrestrial predation is zero or if the colony site never dries up, none

7 of the strategies are evolutionarily stable. The evolutionary stability of the strategy does

8 not depend on the rate of aerial predation, the length of incubation or the laying date

9 distribution.

10

Model 2: with active defence

14 NCS is evolutionarily stable if the following inequality is fulfilled (table 3 left part).

$$
\int_{a}^{l+t_{b}} \frac{k_{g}+\left[k_{a}(N)-k_{a}(1)\right]}{n(t)} d t<0
$$

16 and relation $\mathbf{( 7 a )}$ holds if

$$
k_{g}+\left[k_{a}(N)-k_{a}(1)\right]<0
$$

18 In order to qualitatively explore conditions in which NCS is an ESS, $k_{a}$ is assumed to

19 be $\mathrm{N}$-dependent with the form:

$$
k_{a}(N)=k_{\max }\left[\frac{\alpha}{N}+(1-\alpha)\right]
$$


1 where $\alpha$ is the curvature of the function describing the protection efficiency with the

2 number of adult defending the site and $k_{\max }$ is the avian predation rate when only one

3 pair breeds on the site. Hence, the overall predation rate decreased when $\alpha$ increased.

4 Under these assumptions, the inequality (8) reduces to :

$$
k_{g}<\alpha k_{\max }\left(\frac{1}{N-1}\right)
$$

Figure 1 shows the maximum rate of terrestrial predation $k_{g}$ permitting the

7 evolutionary stability of NCS for different levels of $\alpha$ and $k_{\max }$. Qualitatively, an

8 increase in active defence $(\alpha)$ or an increase in maximum rate of aerial predation $\left(k_{\max }\right)$

9 favours the evolutionary stability of NCS since it requires an increase in the rate of

10 terrestrial predation for CS to be invasive.

Fig. 1 approximately here

12

13 The CS is evolutionarily stable if the following inequality is satisfied (Table 3 right 14 part).

$$
-\int_{a}^{l+t_{b}} \frac{k_{a}\left[F\left(t-t_{i}\right)-F\left(t-t_{b}\right]-k_{a}\left[F(t)-F\left(t-t_{i}\right)\right]\right.}{n(t)} d t+\int_{a}^{l+t_{b}} \frac{k_{g}}{w(t)} d t>0
$$

18 To qualitatively study the evolutionary stability of CS, we suppose the cumulative

19 distribution of the laying dates to be of the exponential form:

$$
f(t)=1-\beta \exp ^{-\theta t}
$$


1 where $\theta$ describes the synchronisation of the laying. The active defence and its

2 efficiency are modelled as above.

3 The CS is thus evolutionarily stable if the following inequality is satisfied.

$5 \frac{k_{g}\left(\exp ^{a \theta}-\exp ^{\left(l+t_{b}\right) \theta l}\right)}{\theta N m \beta\left(\exp ^{\theta t_{i}}-1\right)}-\frac{k_{\max } \alpha\left(2 \exp ^{\theta_{i}}-\exp ^{\theta_{b}}-1\right)\left(\exp ^{\left(2 \theta\left(l+t_{b}\right)\right)}-\exp ^{2 a \theta}\right)}{2 \theta N^{2} m^{2} \beta^{2}\left(\exp ^{\theta_{b}}-1\right)\left(\exp ^{\theta_{i}}-\exp ^{\theta t_{b}}\right)\left(\exp ^{\theta_{i}}-1\right)}>0$

6

7 Figure 2 shows in which conditions CS is evolutionary stable for some usual population

8 parameters of larids. The main results are that the stability of CS depends on the relative

9 levels of terrestrial $\left(k_{g}\right)$ and aerial predation $\left(k_{\max } \alpha\right)$ and in a more complex way on the

10 synchronisation of laying dates $(\theta)$. In the range of biologically realistic parameters, CS

11 is frequently evolutionary stable. CS is invasible by NCS only if the population is

12 submitted to a high rate of realised aerial predation relatively to the terrestrial one and

13 shows at the same time a highly synchronised laying (figure 2).

Fig. 2 approximately here

We only developed here the equations of fecundity computed for individuals

17 whose chicks hatched before the site became accessible to the terrestrial predation.

18 When the chicks hatch after the site became accessible, they progressively reach the

19 "safe" site. Thus until they hatch, the late laid eggs experience exactly the same

20 predation as the non-crèching eggs. Thus, if the inequality satisfying the evolutionary

21 stability are slightly different, the qualitative results are of the same form and our

22 conclusions hold for the two different situations. 


\section{DISCUSSION}

2

3 Three main conclusions can be derived from our results: first the crèching strategy can

4 simply evolve from a non-crèching strategy if the probability for the site to become

5 accessible to terrestrial predation is high. Second, in a species with no anti-predator

6 behaviour, the crèching strategy always invades and is stable in a large range of

7 ecological situations. Third, when individuals actively defend their chicks against

8 terrestrial predation, crèching and non-crèching strategy may both be ESS but remain

9 invasible depending on the predation rates, the efficiency of the defence against

10 predators and the synchronisation of laying.

11 In all cases, evolutionary stability is strongly influenced by the relative rates of

12 aerial and terrestrial predation. The non-crèching strategy is favoured and generally the

13 ESS if the aerial predation is high compared to terrestrial predation while crèching

14 strategy is favoured and is the ESS when terrestrial predation is high relative to aerial

15 predation. Since gulls generally occupy sites surrounded by water (del Hoyo et al.

16 1996), they are protected against the terrestrial predators and crèching strategy cannot

17 evolve from a non-crèching strategy. However, in lagoons, where drying is frequent

18 during the breeding season, terrestrial predation remains possible and is even highly

19 probable over the course of the breeding season. This result confirms our original

20 hypothesis that habitat instability associated with terrestrial predation could exert a

21 strong selective pressure in favour of the crèching behaviour.

22 It has been shown that crèching species show little aggression against the

23 predators of their eggs and chicks (Beer 1966, Isenmann 1976, Veen 1977, Zubakin

24 1985). We demonstrated in this paper that when no anti-predator behaviours exists, the 
1 crèching strategy eventually converges. When a species has a reduced but not null

2 aggressiveness against predators, the crèching strategy is no more convergent but is still

3 invasive and evolutionary stable in many ecological contexts (in particular when

4 terrestrial predation is not null). A little aggressive species could maintain the non-

5 crèching strategy only under a very low rate of terrestrial predation. The non-crèching

6 strategy could thus be maintained while being weakly aggressive only in highly stable

7 habitats protected against terrestrial predation or species living in habitats where no

8 terrestrial predation occurs. To our knowledge, this is the first demonstration that weak

9 aggressiveness observed in crèching species could be a pre-adaptation to this strategy

10 since it favours its evolution and its stability. Since a little aggressive non-crèching

11 species is extremely likely to evolve to crèching and unlikely to then evolve back to the

12 non-crèching strategy, it can explain why no intermediate stage seems to exist between

13 non-crèching and crèching and why no reversion (evolution from crèching to non-

14 crèching strategy) seems to have occurred in larids (A. Besnard, unpublished results).

15 Bearing in mind that the evolutionary stability of the non-crèching strategy is not

16 conditional to the synchronisation of the laying date, we can reject the hypothesis that

17 the high synchronisation of laying, observed in many crèching species (Beer, 1966;

18 Isenmann, 1976; Zubakin, 1985; Mierauska and Buzun, 1991; Besnard et al., submitted)

19 is a pre-adaptation to the crèching strategy. An extreme synchronisation of laying

20 associated with a high rate of aerial predation favours the invasion of the crèching

21 strategy and thus exerts a selective pressure against the maintenance of crèching

22 behaviour. It can thus be suggested that the high synchronisation observed in several

23 crèching species is a response to a selective pressure other than those studied here. We

24 modelled the crèching strategy as a massive departure from the colony site when it 
1 becomes accessible to terrestrial predation and by a progressive aggregation at the

2 newly colonised site by late-hatched chicks. In the field, it seems that crèche formation

3 is punctual and that late breeders who do not join the crèche often abandon their eggs

4 (J.-D. Lebreton, pers. obs.). Such a constraint against a late laying should exert a strong

5 selective pressure for the synchronisation of laying.

6 In summary, our results thus lead to three main conclusions concerning the

7 evolution of the crèching behaviour in Larids. First they confirm our original hypothesis

8 stipulating that crèching behaviour evolved in unstable habitats associated with a high

9 terrestrial predation. Second, they demonstrate for the first time that a reduced

10 aggressiveness against predators is a pre-adaptation to the evolution of crèching

11 behaviour. Finally, high synchronisation of laying dates does not appear to represent a

12 pre-adaptation to the evolution of the crèching and actually favours its invasibility in

13 species actively defending their chicks.

14 The evolution of the crèching strategy under the selective pressure of two

15 different predation rates (terrestrial and aerial) could have been modelled using simple

16 models by comparing, for instance, the instantaneous rate of death for crèching or non-

17 crèching chicks. However, our model allow for one to more easily incorporate

18 parameters such as the synchronisation of laying and to later build up more complex

19 behaviour of the crèching species than those examined here without changing the

20 philosophy of the modelling. Such a flexibility in our way of modelling should favour

21 its utilisation to examine the evolution of crèching strategy in other bird families

22 submitted to different selective pressures. 


\section{AKNOWLEDGMENTS}

2

3 We thank Kate Lessells for her precious commentaries in our modelling approach and 4 John Thompson for his help on improving english of the manuscript. 


\section{REFERENCES}

Beer, C. G. (1966). Adaptation to nesting habitat in the reproductive behaviour of the Black-billed Gull (Larus bulleri). Ibis 108, 394-410.

Besnard, A., N. Sadoul, P.-A. Crochet, and J.-D. Lebreton. (submitted). Crèching as a reproductive strategy in Larids.

Buckley, F. G., and P. A. Buckley. (1972). The breeding ecology of royal Terns Sterna (Thalasseus) maxima maxima. Ibis 114, 344-359.

Carter, H. R., and K. A. Hobson. (1988). Creching behavior of Brandt's Cormorant chicks. The Condor 90, 395-400.

Danchin, E., and R. H. Wagner. (1997). The evolution of coloniality: the emergence of new perspectives. Trends in Evolution and Ecology 12, 342-347.

del Hoyo, J., A. Elliot, and J. Argatal. (1996). Handbook of the birds of the world. Lynx Edicions, Barcelona.

Evans, R. M. (1984). Some causal and functional correlates of creching in young White Pelicans. Canadian Journal of Zoology 62, 814-819.

Gochfeld, M. (1980). Mechanism and adaptive value of reproductive synchrony in colonial seabirds. in J. Burger, B. L. Olla, and H. E. Winn, editors. Behavior of Marine animals. Plenum Press, New York-London.

Hamilton, W. J. (1971). Geometry for the selfish herd. J. Theor. Biol. 31, 295-311.

Isenmann, P. (1976). Contribution à l'étude de la biologie de la reproduction et de l'étho-écologie du goéland railleur, Larus genei. Ardea 64, 48-61.

Kruuk, H. (1967). Predator and anti-predator behaviour of the Black-headed Gull (Larus ridibundus L.). Behaviour suppl. 11, 1-129. 
Maynard Smith, J. (1982). Evolution and the Theory of Games. Cambridge University Press, London.

Mierauska, P., and V. Buzun. (1991). Competitive Interactions between the Herring Gull L. a cachinnans and the Great Blac-headed Gull L. ichthyaetus at Sivash Lake (South Ukraine). Seevögel 12, 34-35.

Patterson, I. J. (1965). Timing and spacing of broods in the Black-headed gull Larus ridibundus. Ibis 107, 433-459.

Pettingill, J. O. S. (1960). Crèche behavior and individual recognition in colony of Rockhopper Penguins. Wilson Bulletin 72, 213-221.

Schaller, G. B. (1964). Breeding behavior of the White Pelican at Yellowstone Lake, Wyoming. The Condor 66, 3-23.

Sibley, C. G., and J. E. Ahlquist. (1990). Phylogeny and classification of birds. Yale University Press, New Haven, Connecticut, USA.

Siegel-Causey, D., and S. P. Kharitonov. (1991). The evolution of coloniality. Current ornithology 7, 285-330.

Spurr, E. B. (1975). Behavior of the Adélie Penguin. The Condor 77, 272-280.

Tinbergen, N. (1956). On the Functions of territory in gulls. Ibis 98, 401-411.

Tinbergen, N. (1959). Comparative studies on the behaviour of Gulls (Laridae): a progress report. Behaviour 15, 1-70.

Veen, J. (1977). Functional and Causal aspects of nest distribution in colonies of the Sandwich Tern. Behaviour supplement XX, 1-193.

Wittenberger, J. F., and G. L. Hunt. (1985). The adaptive significance of coloniality in birds. Avian Biology 8, 1-78. 
Zubakin, V. A. (1985). Types of coloniality in the family Laridae. Proc. Int. Ornithol. congr. 18, 1250-1252.

Zubakin, W. A., and W. E. Flint. (1980). Okologie und Verhalten der Reliktmöwe (Larus relictus Lönnb.). Beitr. Vogelkd. 26, 253-275. 


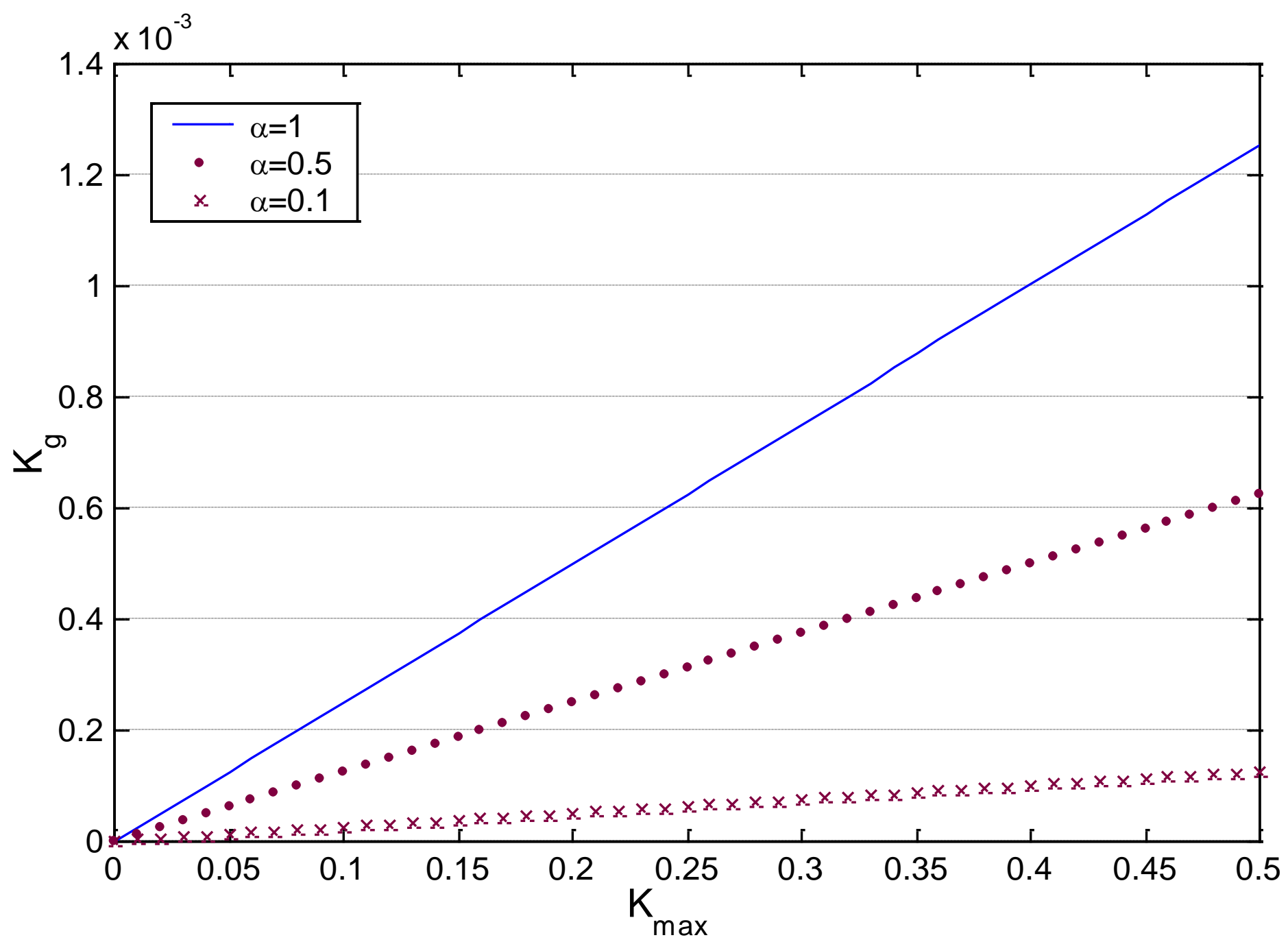

Figure 1 : Maximum rate of terrestrial predation $k_{g}$ permitting the evolutionary stability of NCS for different levels of $\alpha$ and $k_{\max }$. NCS is evolutionarily stable when $k_{g}<\alpha k_{\max }\left(\frac{1}{N-1}\right)$. An increase in active defence $(\alpha)$ or an increase in maximum rate of aerial predation $\left(k_{\max }\right)$ favours the evolutionary stability of NCS since it requires an increase in the rate of terrestrial predation for CS to be invasive. $N$ was fixed to 400 pairs. 


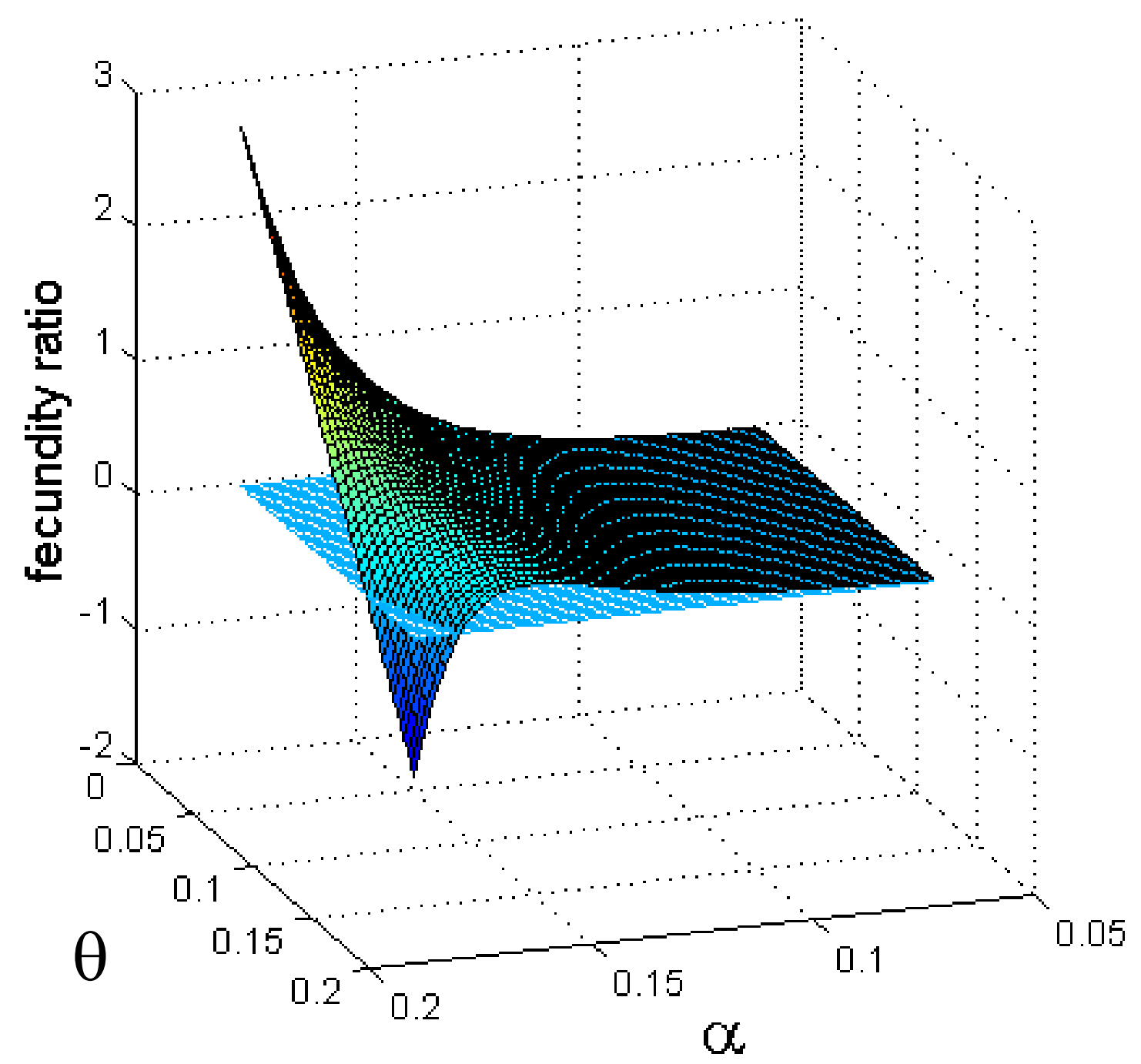

Figure 2 : Conditions in which CS is evolutionarily stable for different levels of synchronisation of laying $(\theta)$ and efficiency of the defence against aerial predators $(\alpha)$. CS is stable when the surface defined by the fecundity ratio is upper than the horizontal plan. The fecundity ratio is given by eq. (12). Parameters in eq. (12) where fixed in order to be biological realistic for gulls: $N=400, m=2, t_{i}=25, t_{b}=30, l=32, a=43$, $K_{g}=0.01, K_{\max }=0.5, \beta=1$. 
Table 1 : Definition of the different parameters used in the ESS modelling approach to model the individual fecundity of crèching or non-crèching birds in population fixed for crèching or noncrèching strategies.

\begin{tabular}{|l|l|}
\hline$F(t)$ & Cumulative distribution function of laying date at date t \\
\hline$N$ & Total number of breeding pairs in the area \\
\hline$m$ & Clutch size \\
\hline$m N F(t)$ & Cumulative number of eggs laid at date $t$ \\
\hline$t_{i}$ & Length of incubation period \\
\hline$t_{r}$ & Length of the rearing period (from hatching to fledging) \\
\hline$t_{b}$ & Total time of rearing from laying to fledging, hence $t_{b}=t_{i}+t_{r}$ \\
\hline$\omega(t)$ & Number of incubated eggs at date $t$ \\
\hline$\pi(t)$ & Number of chicks (i.e. hatched but not yet fledged) at date $t$ \\
\hline$n(t)$ & Total number of egg and chicks, i.e. number of items submitted to predation at date $t$ \\
\hline$k_{a}$ & Rate of aerial predation \\
\hline$k_{g}$ & Rate of ground (terrestrial) predation \\
\hline$l$ & Laying date of an individual \\
\hline$a$ & Date at which the colony site becomes accessible to terrestrial predation \\
\hline
\end{tabular}


Table 2 : Fecundity of the two possible strategies in function of the strategy fixed in the population when no active defence against the predators exists; the fecundity ratio gives the condition of ESS.

\begin{tabular}{|c|c|c|c|}
\hline & & \multicolumn{2}{|c|}{ Population fixed for the strategy } \\
\hline $\begin{array}{c}\text { Rare } \\
\text { Strategy }\end{array}$ & $\begin{array}{c}\text { Non- } \\
\text { Crèching }\end{array}$ & $-\int_{l}^{a} \frac{k_{a}}{n(t)} d t-\int_{a}^{l+t_{b}} \frac{\left(k_{g}+k_{a}\right)}{n(t)} d t$ & $-\int_{l}^{a} \frac{k_{a}}{n(t)} d t-\int_{a}^{l+t_{b}}\left[\frac{k_{a}}{n(t)}+\frac{k_{g}}{\omega(t)}\right] d t$ \\
\cline { 2 - 4 } & Crèching & $-\int_{l}^{l+t_{b}} \frac{k_{a}}{n(t)} d t$ & $-\int_{l}^{l+t_{b}} \frac{k_{a}}{n(t)} d t$ \\
\hline $\begin{array}{c}\text { Condition } \\
\text { satisfying } \\
\text { the ESS }\end{array}$ & $\int_{a}^{l+t b} \frac{k_{g}}{n(t)} d t<0$ & $\int_{a}^{l+t b} \frac{k_{g}}{\omega(t)} d t>0$ \\
\hline
\end{tabular}


Table 3 : Fecundity of the two possible strategies in function of the strategy fixed in the population when an active defence against the predators exists; the fecundity ratio gives the condition of ESS

\begin{tabular}{|c|c|c|c|}
\hline & & \multicolumn{2}{|c|}{ Population fixed for the strategy } \\
\hline \multirow{2}{*}{$\begin{array}{c}\text { Rare } \\
\text { Strategy }\end{array}$} & $\begin{array}{c}\text { Non- } \\
\text { Crèching }\end{array}$ & $-\int_{l}^{a} \frac{k_{a}(N)}{n(t)} d t-\int_{a}^{l+t_{b}} \frac{\left[k_{g}+k_{a}(N)\right]}{n(t)} d t$ & $-\int_{l}^{a} \frac{k_{a}}{n(t)} d t-\int_{a}^{l+t_{b}}\left[\frac{k_{a}\left[F(t)-F\left(t-t_{i}\right)\right]}{n(t)}+\frac{k_{g}}{\omega(t)}\right] d t$ \\
\cline { 2 - 5 } & Crèching & $-\int_{l}^{a} \frac{k_{a}(N)}{n(t)} d t-\int_{a}^{l+t} \frac{k_{a}(1)}{n(t)} d t$ & $-\int_{l}^{a} \frac{k_{a}(N)}{n(t)} d t-\int_{a}^{t l+t_{b}} \frac{k_{a}\left[F\left(t-t_{i}\right)-F\left(t-t_{b}\right)\right]_{d}}{n(t)} d t$ \\
\hline \multirow{2}{*}{$\begin{array}{c}\text { Condition } \\
\text { satisfying } \\
\text { ESS }\end{array}$} & $\int_{a}^{l+t_{b}} \frac{k_{g}+\left[k_{a}(N)-k_{a}(1)\right]}{n(t)} d t<0$ & $-\int_{a}^{l+t_{b}} \frac{k_{a}\left[2 F\left(t-t_{i}\right)-F\left(t-t_{b}\right)-F(t)\right]}{n(t)} d t+\int_{a}^{l+t_{b}} \frac{k_{g}}{w(t)} d t>0$ \\
\hline
\end{tabular}




\section{Appendix A: Derivation of individual fecundity in a system} where adults do not defence the colony site against the predators

(a) Derivation of the fecundity of an individual demonstrating CS in a population fixed for NCS

From (4), the number of offspring at date $t+d t$ is given by :

$$
f(t+d t)=\left[1-\frac{k_{a}}{n(t)} d t\right] f(t)
$$

so that

$$
\frac{f(t+d t)-f(t)}{f(t)}=-\frac{k_{a}}{n(t)} d t
$$

Which leads to

$$
\frac{d \log [f(t)]}{d t}=-\frac{k_{a}}{n(t)} d t
$$

Integrating between $a$ and $l$ both sides of equation (A3) and taking exponential, we obtain the number of offspring at date $a$ of an individual laying at date $l$ :

$$
f(a)_{N C / N C}=f(l) e^{-\int_{l}^{a} \frac{k_{a}}{n(t)} d t}
$$

where NC means Non-Crèching.

After the site became accessible to terrestrial predators, the offspring is submitted to the terrestrial predation $k_{g}$ and the aerial predation $k_{a}$. Doing in the same way as above, we obtain :

$$
f\left(l+t_{b}\right)_{N C / N C}=f(a)_{N C / N C} e^{-\int_{a}^{l+t_{b}} \frac{k_{a}+k_{g}}{n(t)} d t}
$$

using (A4) in (A5), we obtain

$$
f\left(l+t_{b}\right)_{N C / N C}=f(l) e^{-\left[\int_{l}^{a} \frac{k_{a}}{n(t)} d t+\int_{a}^{l+t_{b}} \frac{k_{a}+k_{g}}{n(t)}\right]} d t
$$


(b) Derivation of the fecundity of an individual demonstrating CS in a population fixed for NCS.

Here offspring are not submitted to terrestrial predation after $a$ because they leave the site when it became accessible to terrestrial predation. The procedure described above remains valid.

The fecundity is thus given by

$$
f\left(l+t_{b}\right)_{C / N C}=f(l) e^{-\int_{l}^{l+t_{b}} \frac{k_{a}}{n(t)} d t}
$$

where $\mathrm{C}$ means Crèching and NC means Non-Crèching.

The ratio of the fecundity satisfying the ESS of NCS is given by

$$
\frac{f\left(l+t_{b}\right)_{N C / N C}}{f\left(l+t_{b}\right)_{C / N C}}>1
$$

Using (A6) and (A7), the inequality (A8) is reduced to:

$$
\int_{a}^{l+t_{b}}-\frac{k_{g}}{n(t)} d t>0
$$


(c) Derivation of the fecundity of an individual demonstrating NCS in a population fixed for CS.

Since many CS birds leave the site when it becomes accessible to terrestrial predators, the number of items on the colony sites submitted to terrestrial predation is given by the number of eggs still unhatched. The number of items submitted to aerial predation is still given by the total number of items on both sites.

We thus obtain :

$$
f\left(l+t_{b}\right)_{N C / C}=f(l) e^{\int_{l}^{a}-\frac{k_{a}}{n(t)} d t+\int_{a}^{l+t} b-\left[\frac{k_{a}}{n(t)}+\frac{k_{g}}{\omega(t)}\right] d t}
$$

(d) Derivation of the fecundity of an individual demonstrating CS in a population fixed for CS.

Chicks leave the site when it becomes accessible to terrestrial predators. Thus, they only experience the aerial predation diluted in the whole population of eggs and chicks.

Taking into account what it precedes,

$$
f\left(l+t_{b}\right)_{C / C}=f(l) e^{-\int_{l}^{l+t_{b}} \frac{k_{a}}{n(t)} d t}
$$

Then the fecundity satisfies the ESS of CS provided that

$$
\int_{a}^{l+t} b \frac{k g}{\omega(t)} d t>0
$$




\section{Appendix B: Derivation of individual fecundity in a system where adults actively defence the colony site against the predators}

(a) Derivation of the fecundity of an individual demonstrating CS in a population fixed for NCS.

$$
f\left(l+t_{b}\right)_{N C l N C}=f(l) e^{-\int_{l}^{a} \frac{k_{a}(N)}{n(t)} d t-\int_{a}^{l+t_{b}} \frac{k_{g}+k_{a}(N)}{n(t)} d t}
$$

(b) Derivation of the fecundity of an individual demonstrating CS in a population fixed for NCS.

The chicks of the CS invader are defended only by their own parents on the site they reached after their birth site became accessible to terrestrial predators. The fecundity of an CS in a NCS is thus given by:

$$
f\left(l+t_{b}\right)_{C / N C}=f(l) e^{-\int_{l}^{a} \frac{k_{a}(N)}{n(t)} d t-\int_{a}^{l+t_{b}} \frac{k_{a}(1)}{n(t)} d t}
$$

Then the fecundity ratio satisfying the ESS of NCS is given by

$$
\int_{a}^{l+t_{b}} \frac{k_{g}+k_{a}(N)-k_{a}(1)}{n(t)} d t<0
$$

(c) Derivation of the fecundity of an individual demonstrating NCS in a population fixed for CS.

When the site becomes accessible to terrestrial predators, chicks already hatched leave the colony site. It remains on the colony sites all the eggs still unhatched and protected by their parents. The fecundity is thus given by:

$$
f\left(l+t_{b}\right)_{N C / C}=f(l) e^{-\int_{l}^{a} \frac{k_{a}}{n(t)} d t-\int_{a}^{l+t_{b}}\left[\frac{k_{a}\left[F(t)-F\left(t-t_{i}\right)\right]}{n(t)}+\frac{k_{g}}{\omega(t)}\right] d t}
$$


(d) Derivation of the fecundity of an individual demonstrating CS in a population fixed for CS.

All the parents of the crèching chicks defend the new colonised site simultaneously protected against the terrestrial predation, the fecundity is thus given by:

$$
f\left(l+t_{b}\right)_{C / C}=f(l) e^{-\int_{l}^{a} \frac{k_{a}(N)}{n(t)} d t-\int_{a}^{t l+t_{b}} \frac{k_{a}\left[F\left(t-t_{i}\right)-F\left(t-t_{b}\right)\right]}{n(t)} d t}
$$

Then the fecundity ratio satisfying the ESS of CS is given by:

$$
-\int_{a}^{l+t_{b}} \frac{k_{a}\left[2 F\left(t-t_{i}\right)-F\left(t-t_{b}\right)-F(t)\right]}{n(t)} d t+\int_{a}^{l+t} b \frac{k_{g}}{w(t)} d t>0
$$

